\section{Microbiological profile of chick- en carcasses: A comparative analysis using shotgun metagenomic sequencing}

\author{
Alessandra De Cesare, Federica Palma, \\ Alex Lucchi, Frederique Pasquali, \\ Gerardo Manfreda \\ Department of Agriculture and Food \\ Sciences, Alma Mater Studiorum \\ University of Bologna, Italy
}

\begin{abstract}
In the last few years metagenomic and 16S rRNA sequencing have completly changed the microbiological investigations of food products. In this preliminary study, the microbiological profile of chicken carcasses collected from animals fed with different diets were tested by using shotgun metagenomic sequencing. A total of 15 carcasses have been collected at the slaughetrhouse at the end of the refrigeration tunnel from chickens reared for 35 days and fed with a control diet $(n=5)$, a diet supplemented with $1500 \mathrm{FTU} / \mathrm{kg}$ of commercial phytase $(n=5)$ and a diet supplemented with $1500 \mathrm{FTU} / \mathrm{kg}$ of commercial phytase and $3 \mathrm{~g} / \mathrm{kg}$ of inositol $(\mathrm{n}=5)$. Ten grams of neck and breast skin were obtained from each carcass and submited to total DNA extraction by using the DNeasy Blood \& Tissue Kit (Qiagen). Sequencing libraries have been prepared by using the Nextera XT DNA Library Preparation Kit (Illumina) and sequenced in a HiScanSQ (Illumina) at $100 \mathrm{bp}$ in paired ends. A number of sequences ranging between 5 and 9 million was obtained for each sample. Sequence analysis showed that Proteobacteria and Firmicutes represented more than $98 \%$ of whole bacterial populations associated to carcass skin in all groups but their abundances were different between groups. Moraxellaceae and other degradative bacteria showed a significantly higher abundance in the control compared to the treated groups. Furthermore, Clostridium perfringens showed a relative frequency of abundance significantly higher in the group fed with phytase and Salmonella enterica in the group fed with phytase plus inositol. The results of this preliminary study showed that metagenome sequencing is suitable to investigate and monitor carcass microbiota in order to detect specific pathogenic and/or degradative populations.
\end{abstract}

\section{Introduction}

Techniques and technologies used for detection and characterization of foodborne pathogens in food products have evolved tremendously over the past several decades (Gracias and McKillip, 2004; Nugen and Baeumner, 2008; Valderrama et al., 2015). Traditional methods for pathogen detection, including microscopy and culture-based analyses, are biased according to the specific culture requirements for most genera and species. Moreover, they do not assess the microbiome at ecological level. More modern approaches, including immunoassays and/or nucleic acid amplification, only allow for detection of single or a few specific pathogen(s) at a time. However, it is well known that changes in the surrounding environment cause stresses on bacterial populations, leading to reorganization of microbial communities, which potentially affects the persistence of foodborne pathogens in the food production chain (Larsen et al., 2014; Pricope et al., 2013). Therefore, the real challenge is to assess the influence that the entire microbial communities have on presence of pathogens in food products.

Within this framework, shotgun metagenomic, which is the study of wholecommunity DNA extracted directly from samples, has been increasingly used in multiple disciplines, particularly as sequencing costs decrease and output increases (Manichanh et al., 2008). When compared to target amplicon metagenomics (e.g., 16S rRNA gene sequencing), shotgun metagenomics provides the potential for both higher resolution identification of organisms (i.e., to the strain level), as well as study of microbial communities without introduction of sequencing bias due to unequal amplification of the target gene (Shah et al., 2011). Although obtaining a complete individual genome from metagenomic sequences is still challenging, it is sufficient to characterize the major functions of the microbial communities, as well as to identify their taxon by assigning to public genome reference databases ( $\mathrm{Li}$ et al., 2014). Overall, the goals for metagenomic analysis are to understand i) community composition/structure, including the taxonomic breakdown and relative abundance of the various species; ii) genic contribution of each member of the community, including number and functional capacity; iii) intra-species or intra-population heterogeneity of the genes (Scholz et al., 2012).

Although there are many opportunities to use metage-nomics tools to support detection of foodborne pathogens from foods and food-associated environments,
Correspondence: Alessandra De Cesare, Dipartimento di Scienze e Tecnologie AgroAlimentari, Università di Bologna, via del Florio 2, 40064 Ozzano dell'Emilia (BO), Italy.

Tel: +39.051.2097853 - Fax: +39.051.2097852

E-mail: alessandra.decesare@unibo.it

Key words: Shotgun Metagenomic Sequencing, microbial profile, chicken carcasses.

Contributions: AD, FeP, FrP, AL data collection; $\mathrm{AD}, \mathrm{GM}$ experimental plan and data analysis; AD, GM manuscript writing, reviewing and references search.

Conflict of interests: the authors declare no potential conflict of interests.

Funding: the work was supported by the EU founded project COMPARE (Grant Agreement $\mathrm{N}^{\circ}$ 643476).

Received for publication: 15 July 2017. Revision received: 5 December 2017.

Accepted for publication: 5 December 2017

This work is licensed under a Creative Commons Attribution-NonCommercial 4.0 International License (CC BY-NC 4.0).

(C) Copyright A. De Cesare et al., 2018 Licensee PAGEPress, Italy

Italian Journal of Food Safety 2018; 7:6923 doi:10.4081/ijfs.2018.6923

most meta-genomics studies on the detection of microbes in foods have focused on characterizing the microbial ecology and micro-bial successions during fermentations (van Hijum et al., 2013). The opportunities for metagenomics approaches to improve foodborne pathogen detection are illustrated by a study that used metagenomics approaches to characterize the species composition associated with the tomato phyllosphere - both on the native plant and in the pre-enrichment and enrichment media used to isolate Salmonella (Ottersen et al., 2013). This study was conducted because isolation of Salmonella from the tomato phyllosphere has previously proven challenging despite the fact that tomatoes have been implicated as the source of several human salmonellosis outbreaks. Interestingly, this metagenomic study identified considerable growth of Paenibacillus spp. during enrichment; this is important because this organism may outcompete or even kill Salmonella during enrichment. In addition, sequences matching different Salmonella serovars were identified both from the uncultured samples as well as from different enrichments, sug- 
gesting the presence of Salmonella despite the fact that these samples were negative by both bacteriological methods and real-time PCR. Although these findings do support the possibility that Paenibacillus may have outcompeted Salmonella during enrichment, it is also possible that the detection of Salmonella DNA sequences is due to presence of dead Salmonella cells (Bergholz et al., 2014).

The administration of feed additives in chickens has been linked to changes in the animal gut influencing meat safety. Lactobacillus administration has been shown to reduce colonization by foodborne pathogens like Campylobacter (Ghareeb et al., 2012; Neal-McKinnet et al., 2012), Clostridium (La Ragione et al., 2004) and Salmonella (Chen et al., 2012) improving the microbial food safety of poultry meat. Beside probiotics, broiler diet can be supplemented with enzymes like phytase. Other than making the phosphorus available for the host, the supplementation of phytase can avoid the anti-nutritional effect of phytic acid reducing endogenous losses and increasing protein digestibility.

Since the effect of phytase supplementation on microbiological profile of poultry meat has been never investigated, in this preliminary study shotgun metagenomics has been applied to compare the microbial compositions of 15 chicken carcasses collected at the end of the rearing period (i.e., 35 days) from animals fed with a control diet and diets supplemented with 1500 FTU/kg of commercial phytase and 1500 FTU/kg of commercial phytase plus $3 \mathrm{~g} / \mathrm{kg}$ of inositol.

\section{Materials and Methods}

A total of 15 poultry carcasses were randomly collected at the slaughterhouse at the end of the refrigeration tunnel. All carcasses were obtained from birds belonging to the same breeder flock and hatching session, housed in the same poultry house at the stocking density of about 10 chicks $/ \mathrm{m}^{2}$ and fed with three different diets up to 35 days. A total of 5 carcasses were obtained from birds fed with a basal diet (group A); 5 carcasses from birds fed with the basal diet supplemented with phytase at the concentration of $1500 \mathrm{FTU} / \mathrm{kg}$ feed (group B); 5 carcasses from birds fed with the basal diet supplemented with phytase at the concentration of $1500 \mathrm{FTU} / \mathrm{kg}$ feed and $3 \mathrm{~g} / \mathrm{kg}$ inositol (group C). According to the official sampling procedures to verify the hygienic quality of broiler carcasses, ten grams of neck and breast skin were collected from each carcass and placed in a sterile bag with $40 \mathrm{~mL}$ of sterile saline solution. After homogenization for 1 minute using the Pulsifier $^{\circledR}$ (Microgen Bioproducts Ltd, Cambrige, UK) the whole rinse fluid was

Table 1. Mean relative frequency of abundance (\%) and standard deviation (sd) of phyla, classes and families of skin microbiota in chickens belonging to control group (A), group fed with phytase (group B) and phytase plus inositol (group C).

\begin{tabular}{|c|c|c|c|c|c|c|c|c|}
\hline \multirow[t]{2}{*}{ Phylum } & \multirow[t]{2}{*}{ Class } & \multirow[t]{2}{*}{ Family } & \multicolumn{2}{|c|}{ Group A } & \multicolumn{2}{|c|}{ Group B } & \multicolumn{2}{|c|}{ Group C } \\
\hline & & & Mean & sd & Mean & sd & Mean & sd \\
\hline \multirow[t]{14}{*}{ Proteobacteria } & \multirow{10}{*}{ Gammaproteobacteria } & & 94.99 & 2.60 & 92.68 & 1.42 & 92.81 & 3.25 \\
\hline & & & 94.61 & 2.61 & 92.15 & 1.45 & 92.51 & 3.28 \\
\hline & & Enterobacteriaceae & 22.84 & 11.83 & 42.00 & 15.08 & 37.56 & 5.58 \\
\hline & & Moraxellaceae & 51.36 & 10.17 & 23.71 & 8.59 & 22.05 & 1.48 \\
\hline & & Aeromonadaceae & 19.48 & 3.76 & 25.18 & 5.99 & 30.95 & 9.89 \\
\hline & & Shewanellaceae & 0.47 & 0.15 & 0.82 & 0.38 & 1.48 & 0.48 \\
\hline & & Pseudomonadaceae & 0.31 & 0.04 & 0.30 & 0.11 & 0.33 & 0.08 \\
\hline & & Idiomarinaceae & 0.03 & 0.01 & 0.02 & $<0.01$ & 0.01 & $<0.01$ \\
\hline & & Pasteurellaceae & 0.07 & 0.06 & 0.05 & 0.05 & 0.03 & 0.01 \\
\hline & & Vibrionaceae & 0.02 & $<0.01$ & 0.02 & $<0.01$ & 0.03 & $<0.01$ \\
\hline & \multirow[t]{3}{*}{ Betaproteobacteria } & & 0.35 & 0.08 & 0.51 & 0.23 & 0.26 & 0.09 \\
\hline & & Comamonadaceae & 0.29 & 0.08 & 0.45 & 0.21 & 0.19 & 0.06 \\
\hline & & Neisseriaceae & 0.04 & 0.01 & 0.02 & 0.01 & 0.03 & 0.02 \\
\hline & \multicolumn{2}{|l|}{ Alphaproteobacteria } & 0.02 & $<0.01$ & 0.02 & $<0.01$ & 0.03 & $<0.01$ \\
\hline \multirow[t]{12}{*}{ Firmicutes } & \multirow{9}{*}{ Bacilli } & & 4.01 & 1.38 & 6.46 & 2.12 & 6.10 & 1.84 \\
\hline & & & 3.14 & 1.57 & 3.21 & 1.40 & 3.42 & 0.96 \\
\hline & & Planococcaceae & 0.57 & 0.28 & 0.68 & 0.16 & 0.36 & 0.07 \\
\hline & & Bacillaceae & 0.24 & 0.17 & 0.11 & 0.05 & 0.08 & 0.02 \\
\hline & & Paenibacillaceae & 0.11 & 0.03 & 0.06 & 0.01 & 0.06 & 0.01 \\
\hline & & Staphylococcaceae & 0.19 & 0.09 & 0.56 & 0.71 & 0.18 & $<0.01$ \\
\hline & & Enterococcaceae & 0.33 & 0.30 & 0.45 & 0.16 & 1.17 & 0.62 \\
\hline & & Lactobacillaceae & 0.02 & 0.01 & 0.38 & 0.47 & 0.46 & 0.49 \\
\hline & & Streptococcaceae & 1.66 & 0.97 & 0.96 & 0.67 & 1.09 & 0.39 \\
\hline & \multirow[t]{3}{*}{ Clostridia } & & 0.85 & 1.04 & 3.24 & 1.02 & 2.67 & 2.71 \\
\hline & & Clostridiaceae & 0.77 & 0.97 & 3.18 & 1.03 & 2.61 & 2.69 \\
\hline & & Peptostreptococcaceae & 0.05 & 0.05 & 0.03 & 0.03 & 0.02 & $<0.01$ \\
\hline \multirow[t]{5}{*}{ Bacteroidetes } & & & 0.96 & 0.21 & 0.79 & 0.63 & 0.97 & 0.41 \\
\hline & Bacteroidia & & 0.05 & 0.01 & 0.23 & 0.23 & 0.12 & 0.04 \\
\hline & \multirow{3}{*}{ Flavobacteriia } & Bacteroidaceae & 0.04 & 0.01 & 0.21 & 0.21 & 0.09 & 0.02 \\
\hline & & & 0.90 & 0.20 & 0.55 & 0.10 & 0.83 & 0.39 \\
\hline & & Flavobacteriaceae & 0.90 & 0.20 & 0.55 & 0.10 & 0.83 & 0.39 \\
\hline \multirow[t]{4}{*}{ Actinobacteria } & \multirow{4}{*}{ Actinobacteria } & & 0.04 & 0.04 & 0.06 & 0.02 & 0.10 & 0.05 \\
\hline & & & 0.04 & 0.01 & 0.06 & 0.02 & 0.10 & 0.05 \\
\hline & & Micrococcaceae & 0.01 & $<0.01$ & 0.03 & 0.03 & 0.02 & 0.02 \\
\hline & & Bifidobacteriaceae & 0.003 & $<0.01$ & 0.004 & $<0.01$ & 0.03 & $<0.01$ \\
\hline
\end{tabular}


placed in a $50-\mathrm{mL}$ falcon tube and centrifuged at $6500 \mathrm{x}$ g for 20 minutes at $4^{\circ} \mathrm{C}$ to pellet bacteria.

A total of $0.25 \mathrm{~g}$ of pellet were suspended in $1 \mathrm{~mL}$ lysis buffer $(500 \mathrm{mM} \mathrm{NaCl}, 50$ $\mathrm{mM}$ Tris-Cl, $\mathrm{pH}$ 8.0, $50 \mathrm{mM}$ EDTA, 4\% SDS) with MagNA Lyser Green Beads (Roche, Milan, Italy) and homogenized on the MagNA Lyser (Roche) for $25 \mathrm{sec}$ at $6500 \mathrm{rpm}$. The samples were then heated at $70^{\circ} \mathrm{C}$ for $15 \mathrm{~min}$, followed by centrifugation to separate the DNA from the bacterial cellular debris. The samples were then subjected to $10 \mathrm{M} \mathrm{v} / \mathrm{v}$ ammonium acetate (Sigma, Milan, Italy) precipitation, followed by isopropanol (Sigma) precipitation and a $70 \%$ ethanol (Carlo Erba, Milan, Italy) wash and re-suspended in $100 \mu \mathrm{L} 1 \mathrm{X}$ Tris-EDTA (Sigma). The samples were treated with DNase-free RNase (Roche) and incubated overnight at $4^{\circ} \mathrm{C}$, before being processed through the QIAmp ${ }^{\circledR}$ DNeasy Blood \& Tissue Kit (Qiagen, Milan, Italy) according to manufacturer's directions.

The DNA extracted from each sample was quantified on a BioSpectrometer ${ }^{\circledR}$ (Eppendorf, Milan, Italy) and submitted to library preparation procedure with the Nextera XT DNA Library Preparation Kit (Illumina, San Diego, CA). A total of $5 \mu \mathrm{L}$ of each library (1.3-2 nM) were pooled together and sequenced in the same flow cell using the HiScanSQ sequencer (Illumina) at $100 \mathrm{bp}$ in paired-end mode. Metagenomic sequencing yielded an average of 6.841 million mapped reads/sample, with a Phread quality score always higher than 30. The trimming process was performed to filter all reads shorter than $50 \mathrm{bp}$ and to discharge the trimmed paired-end reads shorter than $50 \mathrm{bp}$. The suitable reads were then mapped to reference sequence databases using MG-RAST.

Results regarding relative frequency of abundances of bacterial taxa at different taxonomic level were compared through the
White's non-parametric t-test, using Statistical Analysis of Metagenomic profile Software v 2.0.9 (STAMP) (Parks et al., 2014). After removing non-bacterial species, taxa abundances were normalized so that each sample total abundance resulted one. Relative frequencies of abundance showing $\mathrm{P}<0.05$ were considered significantly different.

\section{Results}

The microbiota associated to the skin carcasses investigated is summarized in Table 1. Proteobacteria and Firmicutes represented more than $98 \%$ of whole bacterial populations associated to carcass skin in all groups (Table 1). Proteobacteria relative frequency of abundance was higher in the control group in comparison to the groups treated with phytase and phytase plus inositol. On the contrary, Firmicutes abundance

Table 2. Mean relative frequency of abundance (\%) of the 30 top representative species (MRS) of skin microbiota in groups A, B and C.

\begin{tabular}{|c|c|c|c|c|c|c|c|c|c|}
\hline MRS & Group A species & Mean & sd & Group B species & Mean & sd & Group C species & Mean & sd \\
\hline 1 & Acinetobacter johnsonii & 32.86 & 7.07 & Escherichia coli & 36.80 & 15.01 & Escherichia coli & 30.47 & 8.84 \\
\hline 2 & Escherichia coli & 16.27 & 13.04 & Aeromonas veronii & 18.65 & 4.68 & Aeromonas veronii & 22.28 & 6.96 \\
\hline 3 & Aeromonas veronii & 13.83 & 2.43 & Acinetobacter johnsonii & 14.25 & 4.77 & Acinetobacter johnsonii & 14.21 & 0.99 \\
\hline 4 & Acinetobacter lwoffii & 9.84 & 1.99 & Aeromonas hydrophila & 4.46 & 0.97 & Aeromonas hydrophila & 5.77 & 2.10 \\
\hline 5 & Aeromonas hydrophila & 3.78 & 0.87 & Acinetobacter lwoffii & 4.35 & 1.44 & Acinetobacter lwoffii & 4.23 & 0.43 \\
\hline 6 & Citrobacter freundii & 1.32 & 0.36 & Clostridium perfringens & 3.15 & 1.04 & Clostridium perfringens & 2.57 & 2.07 \\
\hline 7 & Aeromonas media & 1.07 & 0.25 & Aeromonas media & 1.28 & 0.26 & Citrobacter freundii & 2.53 & 2.08 \\
\hline 8 & Morganella morganii & 1.05 & 0.84 & Citrobacter freundii & 0.97 & 0.23 & Aeromonas media & 1.67 & 0.57 \\
\hline 9 & Acinetobacter junii & 0.99 & 0.28 & Morganella morganii & 0.74 & 0.54 & Enterococcus cecorum & 0.90 & 0.41 \\
\hline 10 & Acinetobacter towneri & 0.85 & 0.27 & Acinetobacter junii & 0.67 & 0.38 & Shewanella baltica & 0.85 & 0.40 \\
\hline 11 & Acinetobacter baumannii & 0.80 & 0.15 & Kurthia sp. 11 kri321 & 0.66 & 0.16 & Aeromonas salmonicida & 0.81 & 0.35 \\
\hline 12 & Klebsiella oxytoca & 0.74 & 0.20 & Psychrobactersp. P11F6 & 0.52 & 0.13 & Morganella morganii & 0.54 & 0.19 \\
\hline 13 & Clostridium perfringens & 0.73 & 0.35 & Aeromonas salmonicida & 0.46 & 0.08 & Klebsiella oxytoca & 0.45 & 0.12 \\
\hline 14 & Providencia rustigianii & 0.70 & 0.07 & Shewanella baltica & 0.44 & 0.10 & Acinetobacter junii & 0.39 & 0.10 \\
\hline 15 & Streptococcus iniae & 0.67 & 0.30 & Acinetobacter towneri & 0.43 & 0.22 & Streptococcus iniae & 0.38 & 0.10 \\
\hline 16 & Kurthia sp. 11 kri321 & 0.56 & 0.28 & Providencia rustigianii & 0.42 & 0.44 & Empedobacter brevis & 0.37 & 0.08 \\
\hline 17 & Aeromonas salmonicida & 0.53 & 0.37 & Acinetobacter baumannii & 0.40 & 0.15 & Lactobacillus salivarius & 0.35 & 0.08 \\
\hline 18 & Acinetobacter bereziniae & 0.53 & 0.07 & Staphylococcus aureus & 0.37 & 0.27 & Providencia rustigianii & 0.35 & 0.10 \\
\hline 19 & Empedobacter brevis & 0.50 & 0.13 & Streptococcus iniae & 0.36 & 0.28 & Kurthia sp. 11 kri321 & 0.35 & 0.12 \\
\hline 20 & Moraxella bovoculi & 0.49 & 0.43 & Acinetobacter bouvetii & 0.36 & 0.09 & Acinetobacter baumannii & 0.34 & 0.09 \\
\hline 21 & Acinetobacter bouvetii & 0.48 & 0.38 & Klebsiella oxytoca & 0.35 & 0.12 & Acinetobacter bouvetii & 0.29 & 0.02 \\
\hline 22 & Streptococcus dysgalactiae & 0.43 & 0.41 & Lactobacillus salivarius & 0.35 & 0.11 & Streptococcus parauberis & 0.29 & 0.03 \\
\hline 23 & Acinetobacter gerneri & 0.41 & 0.10 & Acinetobacter bereziniae & 0.30 & 0.12 & Citrobactersp.FDAARGOS_156 & 0.25 & 0.04 \\
\hline 24 & Acinetobacter haemolyticus & 0.39 & 0.08 & Comamonas aquatica & 0.29 & 0.07 & Hafnia alvei & 0.24 & 0.07 \\
\hline 25 & Acinetobacter sp. TTH0-4 & 0.39 & 0.07 & Empedobacter brevis & 0.27 & 0.06 & Acinetobacter bereziniae & 0.24 & 0.06 \\
\hline 26 & Acinetobacter venetianus & 0.38 & 0.08 & Klebsiella pneumoniae & 0.26 & 0.07 & Aeromonas molluscorum & 0.24 & 0.06 \\
\hline 27 & Acinetobacter radioresistens & 0.37 & 0.08 & Salmonella enterica & 0.25 & 0.07 & Salmonella enterica & 0.23 & 0.05 \\
\hline 28 & Acinetobacter tandoii & 0.37 & 0.06 & Enterococcus cecorum & 0.24 & 0.07 & Acinetobacter towneri & 0.22 & 0.05 \\
\hline 29 & Acinetobacter parvus & 0.32 & 0.03 & Acinetobacter gerneri & 0.21 & 0.06 & Proteus mirabilis & 0.21 & 0.07 \\
\hline 30 & Enterobacter cloacae & 0.27 & 0.04 & Proteus mirabilis & 0.21 & 0.06 & Klebsiella pneumoniae & 0.21 & 0.05 \\
\hline
\end{tabular}


was higher in the treated groups in comparison to the control. However, those differences were not significantly different.

Gammaproteobacteria was the most abundant class within Proteobacteria, followed by Betaproteobacteria and Alphaproteobacteria (Table 1). Enterococcaceae was the most represented family within Proteobacteria class, followed by Moraxellaceae and Aeromonadaceae (Table 1). Enterococcaceae showed a significantly lower relative frequency of abundance in the control group (22.84\%) in comparison to groups treated with phytase $(42 \%)$ and phytase plus inositol $(37.56 \%)(\mathrm{P}<0.05)$. On the contrary, Moraxellaceae showed a significantly higher abundance in the control $(51.36 \%)$ compared to the treated groups (23.71 and $22.05 \%$, respectively) (i.e., $\mathrm{P}<0.05$ ).

Firmicutes phylum was mostly represented by Bacilli and Clostridia. Within the Bacilli class, the most represented families were Streptococcaceae, Enterococcaceae and Planococcaceae (Table 1). The Enterococcaceae relative frequency of abundance was significantly higher in the group treated with phytase plus inositol in comparison to control group and group treated with phytase only $(\mathrm{P}<0.05)$. On the contrary, Planococcaceae relative frequency of abundance was significantly lower in the group treated with phytase and inositol in comparison to the other groups $(\mathrm{P}<0.05)$ (Table 1). Concerning Clostridia class, both Clostridia $(\mathrm{P}<0.05)$ and Clostridiaceae $(\mathrm{P}<0.05)$ were significantly more abundant in the groups treated with phytase compared to the control.

Within the phylum Bacteroidetes, Flavobacteriia and Flavobacteriaceae showed a significantly lower frequency of abundance in the group treated with phytase only in comparison to the control group $(\mathrm{P}<0.05)$ and the group fed with phytase and inositol $(\mathrm{P}<0.05)$. On the contrary Bacteroidia and Bacteroidaceae showed a relative frequency of abundance significantly higher in the group treated with phytase only compared to other groups $(\mathrm{P}<0.05)$ (Table 1). Finally, phylum Actinobacteria showed a relative frequency of abundance significantly higher $(\mathrm{P}<0.05)$ in the control group in comparison to treated groups $(\mathrm{P}<0.05)$. Moreover, in the group treated with phytase and inositol, Bifidobacteriaceae showed a significantly higher abundance in comparison to the control group $(\mathrm{P}<0.05)$ and group treated with phytase only $(\mathrm{P}<0.05)$ (Table 1$)$.

Table 2 summarizes the top 30 bacterial species, in terms of abundance, identified in the investigated groups. The control group showed a dominance of Acinetobacter and Aeromonas genera, while the treated groups showed a more diversified bacterial population (Table 2). Treated groups presented higher relative frequency of abundances of Escherichia coli compared to the control group (Table 2). On the contrary, Clostridium perfringens showed a relative frequency of abundance significantly lower in the control group in comparison to the group fed with phytase only (Table 3 ). Moreover, Salmonella enterica, representing, one of the top 30 species in both treated groups, was significantly higher in the carcasses collected from birds fed with phytase and inositol in comparison to the control (Table 4). Concerning other foodborne pathogens, Aeromonas hydrophila and

Table 3. Statistically significant differences between means of relative frequency of abundance (\%) of skin bacterial species in control group (A) and group fed with phytase (B).

\begin{tabular}{|c|c|c|c|}
\hline Species & Group A, mean & Group B, mean & P values \\
\hline Acinetobacter johnsonii & 32.86 & 14.25 & 0.0024 \\
\hline Acinetobacter lwoffii & 9.84 & 4.35 & 0.0021 \\
\hline Acinetobacter towneri & 0.85 & 0.43 & 0.0435 \\
\hline Acinetobacter baumannii & 0.80 & 0.40 & 0.0059 \\
\hline Klebsiella oxytoca & 0.74 & 0.35 & 0.0098 \\
\hline Clostridium perfringens & 0.73 & 3.15 & 0.0088 \\
\hline Acinetobacter bereziniae & 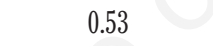 & 0.30 & 0.0121 \\
\hline Empedobacter brevis & 0.50 & 0.27 & 0.0134 \\
\hline Acinetobacter gerneri & 0.41 & 0.21 & 0.0340 \\
\hline Acinetobacter haemolyticus & 0.39 & 0.20 & 0.0090 \\
\hline Acinetobacter sp. TTH0-4 & 0.39 & 0.18 & 0.0023 \\
\hline Acinetobacter venetianus & 0.38 & 0.18 & 0.0069 \\
\hline Acinetobacter radioresistens & 0.37 & 0.20 & 0.0148 \\
\hline Acinetobacter tandoii & 0.37 & 0.17 & 0.0084 \\
\hline Enterobacter cloacae & 0.27 & 0.17 & 0.0072 \\
\hline Enterobacter asburiae & 0.26 & 0.14 & 0.0024 \\
\hline Acinetobacter ursingii & 0.23 & 0.13 & 0.0195 \\
\hline Acinetobacter sp. Ver3 & 0.22 & 0.10 & 0.0155 \\
\hline Acinetobacter pittii & 0.21 & 0.10 & 0.0178 \\
\hline Leclercia adecarboxylata & 0.20 & 0.09 & 0.0022 \\
\hline Moraxella osloensis & 0.15 & 0.09 & 0.0231 \\
\hline Serratia liquefaciens & 0.12 & 0.06 & 0.0025 \\
\hline Acinetobacter bohemicus & 0.11 & 0.05 & 0.0061 \\
\hline Pseudomonas aeruginosa & 0.11 & 0.08 & 0.0457 \\
\hline Paenibacillus sophorae & 0.10 & 0.06 & 0.0322 \\
\hline Citrobacter youngae & 0.10 & 0.07 & 0.0453 \\
\hline Enterobacter sp. 638 & 0.095 & 0.056 & 0.0122 \\
\hline Acinetobacter sp. ATCC 27244 & 0.090 & 0.047 & 0.0236 \\
\hline Bacillus mycoides & 0.079 & 0.035 & 0.0041 \\
\hline Acinetobacter harbinensis & 0.057 & 0.030 & 0.0337 \\
\hline Acinetobacter equi & 0.054 & 0.023 & 0.0034 \\
\hline Pseudomonas pseudoalcaligenes & 0.048 & 0.025 & 0.0025 \\
\hline Chryseobacterium gleum & 0.047 & 0.030 & 0.0457 \\
\hline Acinetobacter sp. NIPH 298 & 0.047 & 0.026 & 0.0428 \\
\hline Acinetobacter nosocomialis & 0.040 & 0.020 & 0.0037 \\
\hline Enterobacter cancerogenus & 0.040 & 0.021 & 0.0016 \\
\hline Citrobacter amalonaticus & 0.025 & 0.018 & 0.0367 \\
\hline Shigella flexneri & 0.017 & 0.043 & 0.0320 \\
\hline Shigella sonnei & 0.016 & 0.043 & 0.0379 \\
\hline Shigella dysenteriae & 0.014 & 0.033 & 0.0238 \\
\hline
\end{tabular}


Aeromonas veronii had a higher relative frequency of abundances in the treated groups in comparison to the control but those differences were not statistically relevant (Table 2). On the contrary Pseudomonas pseudoalcaligenes was significantly higher in the control in comparison to both treated groups (Tables 3 and 4), whereas Pseudomonas aeruginosa was significantly higher in the control in comparison to the group treated with phytase only (Table 3 ). Finally, species belonging to genera Shigella and Shewanella were significantly lower in the control group in comparison to the treated groups (Tables 3 and 4).

\section{Discussion}

The results of this preliminary study showed that the carcass skin microbiota is mainly composed by Proteobacteria and Firmicutes. The same phyla represent more than $95 \%$ of bacterial population in the caeca of one-day old chicks (De Cesare et al., 2017). During the chicken life, the mean relative frequency of abundance of Proteobacteria in the caeca tends to decrease, between $9.61 \%$ at day 1 to $1.74 \%$ at day 41 . On the contrary, the mean relative frequency of abundance of Firmicutes tends to increase, between $85.85 \%$ at day 1 to $93.93 \%$ at day 41 . In the carcasses investigated in this study at the end of slaughtering, the mean relative frequency of abundance of Proteobacteria ranged between 92.68 and $94.99 \%$, whereas that of Firmicutes between 4.01 and $6.46 \%$. Statistically significant differences were determined between bacterial classes and families colonizing carcasses obtained from animals fed with different diets. Enterobacteriaceae were more abundant in the treated groups than in control group and this difference corresponded to a higher abundance of Escherichia coli and Salmonella enterica in carcasses collected from chickens fed with phytase and insositol in comparison to the control. Moreover, Clostridiaceae were significantly higher in the treated groups in comparison to the control and this difference corresponded to a significative higher abundance of Clostridium perfringens in the group fed with phytase in comparison to the control. Differences in contamination of carcasses belonging to control and treated groups might come from the chicken diet but might also come from cross contamination during slaughtering. Beside the origin of the contamination, it is important to note that metagenomics sequencing was able to detect differences even related to low levels of abundance like those associated to
Table 4. Statistically significant differences between means of relative frequency of abundance (\%) of skin bacterial species in control group (A) and group fed with phytase plus inositol (C).

\begin{tabular}{|c|c|c|c|}
\hline Species & Group A, mean & Group C, mean & P values \\
\hline Acinetobacter johnsonii & 32.86 & 14.21 & 0.0008 \\
\hline Acinetobacter lwoffii & 9.84 & 4.23 & 0.0005 \\
\hline Acinetobacter junii & 0.99 & 0.39 & 0.0035 \\
\hline Acinetobacter towneri & 0.85 & 0.22 & 0.0024 \\
\hline Acinetobacter baumannii & 0.80 & 0.34 & 0.0003 \\
\hline Klebsiella oxytoca & 0.74 & 0.45 & 0.0394 \\
\hline Acinetobacter bereziniae & 0.53 & 0.24 & 0.0001 \\
\hline Acinetobacter gerneri & 0.41 & 0.16 & 0.0019 \\
\hline Acinetobacter haemolyticus & 0.39 & 0.18 & 0.0006 \\
\hline Acinetobacter sp. TTH0-4 & 0.39 & 0.17 & 0.0005 \\
\hline Acinetobacter venetianus & 0.38 & 0.17 & 0.0010 \\
\hline Acinetobacter radioresistens & 0.37 & 0.14 & 0.0003 \\
\hline Acinetobacter tandoii & 0.37 & 0.16 & 0.0021 \\
\hline Acinetobacter parvus & 0.32 & 0.18 & 0.0033 \\
\hline Enterobacter cloacae & 0.27 & 0.18 & 0.0014 \\
\hline Acinetobacter schindleri & 0.26 & 0.07 & 0.0228 \\
\hline Enterobacter asburiae & 0.26 & 0.13 & 0.0007 \\
\hline Shewanella baltica & 0.23 & 0.85 & 0.0162 \\
\hline Acinetobacter ursingii & 0.23 & 0.09 & 0.0003 \\
\hline Acinetobacter sp. Ver3 & 0.22 & 0.08 & 0.0017 \\
\hline Acinetobacter pittii & 0.21 & 0.09 & 0.0013 \\
\hline Leclercia adecarboxylata & 0.20 & 0.10 & 0.0027 \\
\hline Comamonas aquatica & 0.19 & 0.10 & 0.0434 \\
\hline Moraxella osloensis & 0.15 & 0.08 & 0.0147 \\
\hline Salmonella enterica & 0.14 & 0.23 & 0.0292 \\
\hline Klebsiella pneumoniae & 0.14 & 0.21 & 0.0015 \\
\hline Serratia liquefaciens & 0.12 & 0.06 & 0.0066 \\
\hline Acinetobacter gyllenbergii & 0.12 & 0.05 & 0.0007 \\
\hline Acinetobacter bohemicus & 0.11 & 0.05 & 0.0015 \\
\hline Paenibacillus sophorae & 0.10 & 0.06 & 0.0403 \\
\hline Acinetobacter sp. ATCC 27244 & 0.09 & 0.04 & 0.0012 \\
\hline Enterococcus cecorum & 0.08 & 0.90 & 0.0222 \\
\hline Bacillus mycoides & 0.08 & 0.03 & 0.0009 \\
\hline Aeromonas schubertii & 0.07 & 0.12 & 0.0471 \\
\hline Enterobacter sp. E20 & 0.07 & 0.03 & 0.0002 \\
\hline Shewanella oneidensis & 0.07 & 0.16 & 0.0002 \\
\hline Acinetobacter harbinensis & 0.06 & 0.02 & 0.0020 \\
\hline Acinetobacter equi & 0.05 & 0.02 & 0.0009 \\
\hline Shewanella putrefaciens & 0.05 & 0.17 & 0.0027 \\
\hline Pseudomonas pseudoalcaligenes & 0.05 & 0.03 & 0.0029 \\
\hline Acinetobacter sp. NIPH 298 & 0.05 & 0.02 & 0.0014 \\
\hline Shewanella sp. ANA-3 & 0.04 & 0.09 & 0.0004 \\
\hline Acinetobacter nosocomialis & 0.04 & 0.02 & 0.0001 \\
\hline Macrococcus caseolyticus & 0.04 & 0.07 & 0.0255 \\
\hline Enterobacter cancerogenus & 0.04 & 0.02 & 0.0022 \\
\hline Plesiomonas shigelloides & 0.04 & 0.01 & 0.0360 \\
\hline Comamonas kerstersii & 0.04 & 0.02 & 0.0284 \\
\hline Bacteroides fragilis & 0.03 & 0.05 & 0.0222 \\
\hline Shewanella sp. MR-7 & 0.03 & 0.06 & 0.0005 \\
\hline Shewanella sp. MR-4 & 0.03 & 0.06 & 0.0003 \\
\hline Edwardsiella tarda & 0.03 & 0.04 & 0.0428 \\
\hline Idiomarina loihiensis & 0.02 & 0.01 & 0.0197 \\
\hline
\end{tabular}


Salmonella enterica. Differences were also quantified in relation to degradative microflora. In fact, Moraxellaceae class resulted significantly more abundant in the control in comparison to treated groups.

Even if the results of this preliminary study support the potential for metagenomics applications in food safety, the use of metagenomics as a tool for the detection of foodborne pathogens in foods still faces several challenges. For one, metagenome sequencing will detect DNA from both dead and alive organisms. An additional challenge is that metagenomics will create massive sequence data sets linked to a given food or food-associated facility (e.g., processing facility or farm), and these are likely to contain at least some sequence data that can easily be misconstrued as indicating a food safety hazard (e.g., the presence of antimicrobial resistance genes or virulence genes). Because, at least in some countries, food safety testing data may have to be released, under specific circumstances, to lawyers or regulatory agencies, some facilities may be reluctant to use these tools out of fear that the data created could inadvertently (and incorrectly) implicate a facility as having evidence of pathogen presence in a food or environment. In addition, data from metagenomic studies of human specimens could potentially be linked to individuals because the data generated may also contain host sequence data that could potentially identify a patient. Both these potential issues may be addressed through initial filtering and removal of sequence data (e.g., human sequences). Future development of guidelines on the proper and ethical use of metagenomics data in food safety may nevertheless be necessary to encourage and facilitate the use of these potentially powerful tools (Bergholz et al., 2014).

\section{Conclusions}

The results of this preliminary study showed that metagenome sequencing is a suitable approach to investigate the microbiota composition of chicken carcasses. Statistically significant differences have been detected between the metagenomes associated to carcasses obtained from chickens fed with different diets. Further studies will clarify if such differences derive from the diets or from other factors and the relationship between abundance of sequencing reads and number of bacterial cells belonging to degradative and pathogenic bacteria.

\section{References}

Bergholz TM, Moreno Switt AI, Wiedmann M, 2014. Omics approaches in food safety: fulfilling the promise ? Trends Microbiol 22:275-81.

Chen CY, Tsen HY, Lin CL, Yu B, Chen CS, 2012. Oral administration of a combination of select lactic acid bacteria strains to reduce the Salmonella invasion and inflammation of broiler chicks. Poultry Sci 91:2139-47.

Gracias KS, McKillip JL, 2004. A review of conventional detection and enumeration methods for pathogenic bacteria in food. Can J Microbiol 50:883-90.

Ghareeb K, Awad WA, Mohnl M, Porta R, Biarnés M, Böhm J, Schatzmayr G, 2012. Evaluating the efficacy of an avian-specific probiotic to reduce the colonization of Campylobacter jejuni in broiler chickens. Poultry Sci 91:182532.

La Ragione RM, Narbad A, Gasson MJ, Woodward MJ, 2004. In vivo characterization of Lactobacillus johnsonii FI9785 for use as a defined competitive exclusion agent against bacterial pathogens in poultry. Lett Appl Microbiol 38:197-05.

Larsen $\mathrm{MH}$, Dalmasso $\mathrm{M}$, Ingmer $\mathrm{H}$, Langsrud S, Malakauskas M, Mader A, Møretrø T, Smole Možina S, Rychli K, Wagner M, John Wallace R, Zentek J, Jordan K, 2014. Persistence of foodborne pathogens and their control in primary and secondary food production chains. Food Control 44: 92-109.

Li J, Jia H, Cai X, Zhong H, Feng Q, Sunagawa S, Arumugan M, 2014. An integrated catalog of reference genes in the human gut microbiome. Nat Biotech 32:834-41.

Manichanh C, Chapple CE, Frangeul L,
Gloux K, Guigo R, Dore J, 2008. A comparison of random sequence reads versus $16 \mathrm{~S}$ rDNA sequences for estimating the biodiversity of a metagenomic library. Nucleic Acids Res 36:5180-88.

Neal-McKinney JM, Lu X, Duong T, Larson CL, Call DR, Shah DH, Konket ME, 2012. Production of organic acids by probiotic lactobacilli can be used to reduce pathogen load in poultry. PLoS One 7:e43928.

Nugen SR, Baeumner AJ, 2008. Trends and opportunities in food pathogen detection. Anal Bioanal Chem 391:451-4.

Ottesen AR, Gonzalez A, Bell R, Arce C, Rideout S, Allard M, Brown E, 2013. Co-enriching microflora associated with culture based methods to detect Salmonella from tomato phyllosphere. PloS One 8:e73079.

Parks DH, Tyson GW, Hugenholtz P, Beiko RG, 2014. STAMP: statistical analysis of taxonomic and functional profiles. Bioinform 30:3123-4.

Pricope L, Nicolau A, Wagner M, Rychli K, 2013. The effect of sub lethal concentrations of benzalkonium chloride on invasiveness and intracellular proliferation of Listeria monocytogenes. Food Control 31:230-35.

Scholz MB, Lo CC, Chain PS, 2012. Next generation sequencing and bioinformatics bottleneck: the current state of metagenomics data analysis. Curr Opin Biotech 23:9-15.

Shah N, Tang H, Doak TG, Ye Y, 2011. Comparing bacterial communities inferred from 16S rRNA gene sequencing and shotgun metagenomics. Pac Symp Biocomput pp 165-76.

Valderrama WB, Dudley EG, Doores S, Cutter CN, 2015. Commercially available rapid methods for detection of selected foodborne pathogens. Crit Rev Food Sci Nutr 56:1519-31.

van Hijum SA, Vaughan EE, Vogel RF, 2013. Application of state-of-art sequencing technologies to indigenous food fermentations. Curr Opin Biotech 24:178-86. 